IOSR Journal of Engineering

May. 2012, Vol. 2(5) pp: 1121-1123

\title{
Automated Safety Systems
}

\author{
Ebinezaru Babu Gollamudi ${ }^{1}$, Kiran Raj Goli ${ }^{2}$, Tippa Bhima Sankara Rao ${ }^{3}$, \\ ${ }^{1}$ Assistant Professor, ${ }^{2}$ B.Tech, ${ }^{3}$ Professor \\ Department of Mechanical Engineering \\ ${ }^{1}$ PACE Institute of Technology \& Sciences, Ongole ${ }^{2}$ PACE Institute of Technology \& Sciences, Ongole \\ ${ }^{3}$ Nimra Institute of Science \& Technology, Vijayawada, Andhra Pradesh, India
}

\begin{abstract}
In this paper, an automated safety system for real time safety is presented. The fields where I am going to use the automated techniques and the simple process is discussed here. The fields are High speed safety, House safety, Industrial safety and mainly the brief description is given for the High speed safety.
\end{abstract}

KEYWORDS: - Actuators, Microprocessors, Sensors, Logic solvers, Timers, Alarms, Carbon plastics etc.

\section{INTRODUCTION}

Many safety systems have been developed and generally they are equipped with alarms and some sort of indicators along with this system, automated safety system has also been developed which is commonly used in large scale industries because of there expensiveness, and my idea is to apply this automated techniques for safety purpose and make it at the possible low cost this is the main aim. The need for this is high that it safe guards humans from the accidental injuries very efficiently. If this is still developed there is a chance for tensionless duty. My intention behind designing this is to make living easy as the modern world demands the same. Fast and accuracy is important but generally in practical fast is possible or accuracy is possible but attaining both at the same time is a little difficult thing, it is made a bit easy using this concepts .The process of my automation can be seen in the block diagram given below

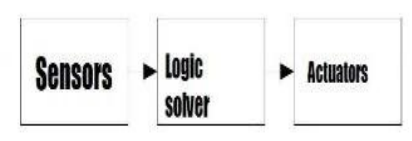

The action for safety must be performed by the actuators and for this sensors are used which are assigned to a pre programmed microcontrollers which sends the signal to actuator connected to it, and it performs the action for safety.

\section{AREA OF MY APPLICATION FOR SAFETY}

\subsection{HIGH SPEED SAFETY}

A driver in a vehicle at a speed of $150 \mathrm{kmp} / \mathrm{h}$ can't stop suddenly by applying breaks if he sees a obstacle in his path suddenly at a distance if about $1 \mathrm{~km}$ from his vehicle so there may be a chance of collision (hitting) but this can be avoided by equipping the following thing, An ultrasonic sensor which has high sensitivity it's range is about $2 \mathrm{~km}$ this is assigned to a microprocessor the micro processor is programmed in such a way that when ever it gets a signal from the ultrasonic sensor if action should be taken then immediately it sends the signal to the actuator Here the actuator is the fuel flow controlling device it controls the flow of fluid from fuel pipe line to entrance of combustion chamber, For this a clipping device is designed, the function of the clipping device is to reduce the crosssectional area of fuel entering the combustion chamber so that low amount of fuel entering the chamber means engine runs at low R.P.M so speed gradually reduces in a short span of time.

Microprocessor: 16-bit

Ultrasonic sensor: 2 in quantity

Speed sensor: 1 in quantity

Range of sensor: 0.5-3.0 kilometers

Response time: 10-30 milli seconds

This device starts working only when the vehicle reaches a stipulated speed i.e. the speed at which the respective vehicle can't stop immediately.

The clipping device is the device which is used to reduce the cross sectional area as stated above and it presses the fuel carrying tube just at the place of entrance i.e. entrance of combustion chamber. The controlled rate of flow of fuel at the time of operation of this device depends on the capacity of the engine, speed at which the vehicle traveling at that instant, and according to that the angle of the limbs of the clipping device is pre arranged so that the fuel flow can be controlled in a desired manner. This must be done cautiously and it must not be neglected because if it just presses fully the fuel carrying pipe line if no fuel reaches 
the combustion chamber there may be a chance of sudden stopping of engine which irritates the driver.

The other important point is the material of the pipe line if it is petrol engine then no problem because it is having the flexible pipe line at the entrance of combustion chamber fixed to a carburetor, so it can be pressed using this device directly but in case of diesel engine the available pipe line is made of metal and that must be replaced by carbon plastics which is flexible and at the same time it is strong and durable.

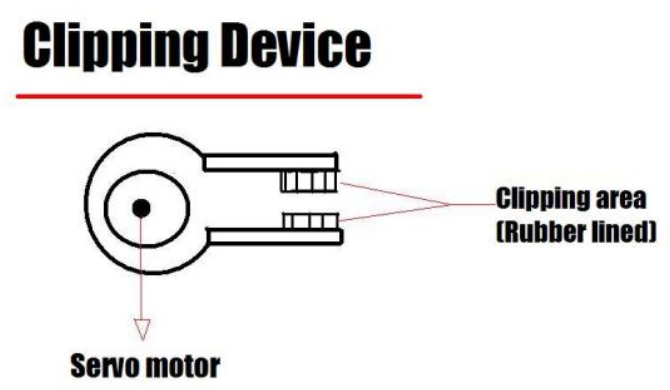

\subsection{HOUSE SAFETY}

Generally for cooking purpose in homes LPG gas cylinders are used if in case of any leakage at the day time the human can sense the smell of that and can open the windows and doors normally so no problem then but in case of night times if any leakage though human senses the smell generally there is a chance of switching ON the light so there is a chance of explosion due to the spark released from the switch which is hazardous so if we equip the LPG gas detecting system which gives a alarm just after the detection human be cautious and no switch would be ON there by eliminating the problem.

\subsection{INDUSTRIAL SAFETY}

In small scale industries like timber cutting and some other cutting industries the cutting machines are equipped for large scale production and in hurry when any part of the human limb gets nearer to the cutting blade portion there may be a chance of injury to any part of the limb of the labourer working there, this can be avoided by equipping a infrared sensor which senses the heat of human body and there by gives the signal to the microprocessor the microprocessor is programmed in such a way that when ever it gets a signal from infrared sensor it gives the signal to the power unit of the cutting machine then the machine cuts off the power thereby stopping of cutting machine and thus avoiding the injury.

\section{APPLICATION OF AUTOMATION IN PRESENT WORLD}

In large scale industries and expensive machine or tools generally we are using this automation [1] techniques and this lead to fast production of goods, and profits of the companies also increased

But at the same time unemployability also increased so there are people who doesn't encourage this technology and it's expensive too. According to my technique this is used for providing safety and it's not complicated also so this can be easily manufactured and usage doesn't require more skill and maintenance. The thing is I am using this technique for only safety purpose so no problem of unemployability which can be considered useful.

\section{PROCESS OF WORKING}

Here the simple things we use are sensors, logic solvers, and actuators the first thing is sensors are different for different areas of detection so the suitable sensor for our application area must be selected then the selection of microprocessor we can select 8-bit, 16-bit, 32-bit according to our needs and then the programming language to the processor must be decided generally languages like 'Embedded $C^{\prime}$ etc are used and now comes the actuator it may be a valve or limb of a machine it depends on the part of the machine which we want to control for the safety

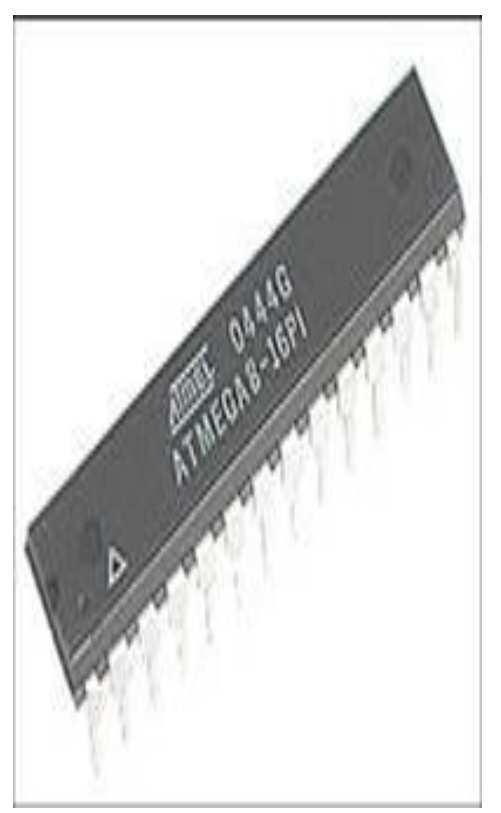



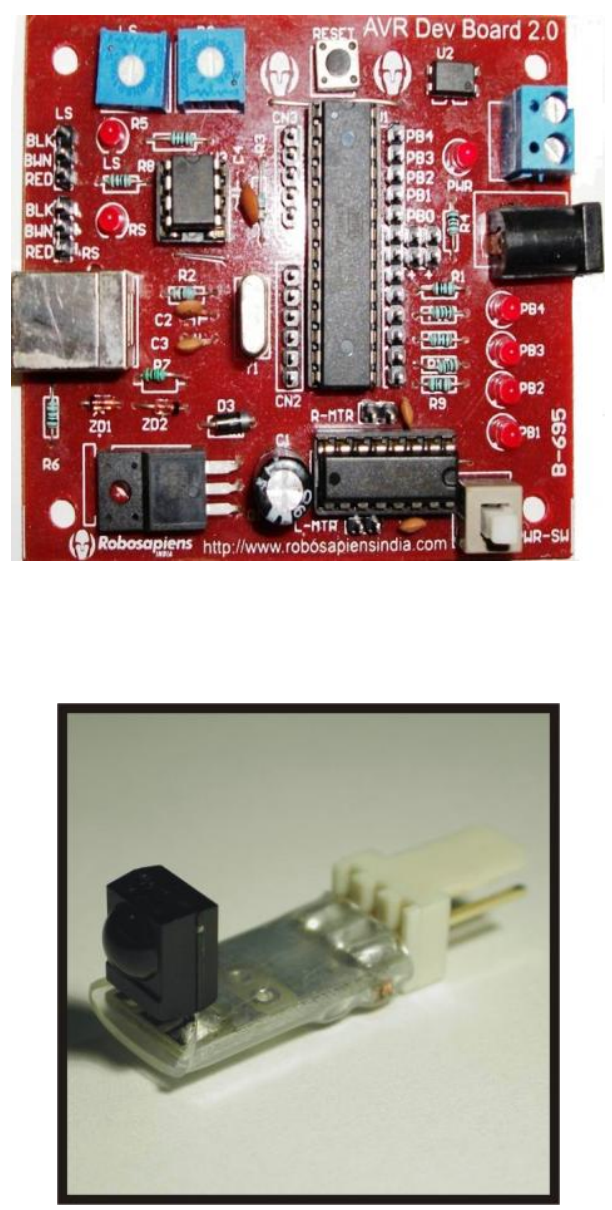

Sensors are equipped for the detection so when ever it detects the change it gives the signal to microprocessor, it is programmed in such a way that it has a ability of taking the decisions so if it thinks the signal is important then it sends the signal to the actuator which is the action performing device here used for providing safety.Here any typical logics are not used and the device can be very simple the thing is selection of appropriate sensor and the microprocessor assigning to it. This is comparatively cheaper then the present automation tools used in the world and it is used for safety purpose thus ensuring tensionless living.

Microprocessor: 16-bit suggested

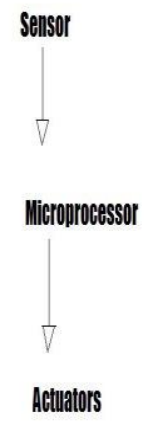

\section{CONCLUSIONS}

This system is designed to ensure the safety for all the possible situations and that too at lowest possible manufacturing cost thus we can ensure that safety is provided for all people effectively and making life tension free.

\section{REFERENCES}

[1]Bijoy K.Gosh, Ning Xi, T.J.Tarn, Control in Robotics and Automation sensor based integration Practical observation and study. 